\title{
Microenvironment-dependent cues trigger miRNA-regulated feedback loop to facilitate the EMT/MET switch
}

\author{
Julienne L. Carstens, Sara Lovisa, and Raghu Kalluri \\ Department of Cancer Biology, Metastasis Research Center, University of Texas MD Anderson Cancer Center, Houston, Texas, USA.
}

\begin{abstract}
The metastatic spread of tumor epithelial cells accounts for over $90 \%$ of cancer-specific mortality; however, the molecular mechanisms that govern tumor spread and distant recolonization remain unclear. In this issue of JCI, Rokavec and colleagues shine light on this murky aspect of tumor biology by focusing through the lens of microenvironmental contributions, namely inflammation, as driving signals that set off a delicate, intracellular feedback loop among cytokine receptors, transcription factors and miRNAs. This study provides in vivo evidence and identifies molecular players behind the elusive switch that drives the epithelial-to-mesenchymal transition and the mesenchymal-to-epithelial transition.
\end{abstract}

\section{EMT/MET switch}

The migratory capacity conferred on cells following the epithelial-to-mesenchymal transition (EMT) is a developmental necessity that has become a predominant hypothesis to explain epithelial cancer metastases and therapeutic resistance (1). In the simplest terms, selective pressures (e.g., nutrient deprivation, hypoxia, therapeutics, etc.) on epithelial tumor cells activate dormant signaling pathways that allow these tumor cells to lose epithelial characteristics and take on mesenchymal properties, including migration and invasion that are advantageous for tumor survival. The concept that EMT promotes cancer metastasis is supported by the reality that mesenchymal tumors, such as sarcomas, are some of the most difficult tumors to treat. However, if mesenchymal properties are so advantageous for cancer spread, why then do most metastatic lesions display epithelial properties similar to those of the primary tumor?

A predominant answer to this question resides in the mesenchymal-to-epithelial transition (MET), which would allow transformed mesenchymal-like cells to regain their epithelial characteristics, a state that is perhaps more advantageous for survival in a foreign microenvironment. As obvious as this explanation

Conflict of interest: The authors have declared that no conflict of interest exists.

Citation for this article: J Clin Invest. 2014; 124(4):1458-1460. doi:10.1172/JCI75239. appears to be, there are many unanswered questions that remain. For instance, there is a lack of solid in vivo evidence to support the induction of MET following metastasis. Furthermore, if MET does occur, what are the molecular pathways involved in promoting tumor aggressiveness (EMT), and how can these pathways be dialed back enough to reestablish epithelial characteristics? Furthermore, what are the unique aspects of the primary and distant microenvironments that provide the necessary stimuli to initiate the switch between EMT and MET?

\section{Primary and distant tumor microenvironments}

Tumor epithelia cells exist within a complex amalgam of fibroblasts, immune cells, nervous cells, vasculature, musculature, soluble signaling molecules, and extracellular matrices commonly referred to as the microenvironment (2). This microenvironment is dynamic and coevolves with the tumor cells to inhibit or further enhance progression depending on its specific components (Figure 1). The primary microenvironment is associated with acute inflammation in response to the presence of the tumor and disruptions in the basement membrane. This inflammation is orchestrated by a complex symphony of cytokine and chemokine signaling molecules. Of these, a highly studied chemokine, IL-6, is secreted by and acts on many cells within the microenvironment and seems to universally promote inflammatory and tumor progression as well as $\operatorname{EMT}(3,4)$. Furthermore, high levels of serum IL- 6 have been correlated with poor survival outcomes. While the local inflammation is critical for tumor development and progression, the role of localized inflammation in the recolonization of tumor cells in a distant microenvironment is still being investigated.

\section{Suppression of miR-34a is required for IL-6-driven EMT}

In this issue of the JCI, Rokavec et al. (5) report that they examined the mechanism of the EMT/MET switch, identified an IL-6-driven feedback loop that allows for the induction of EMT, and determined that removal of IL- 6 signaling subsequently reverses the effects of this feedback loop. Mutations in the gene encoding the tumor suppressor p53 that result in loss of function are the most common mutations in adult solid tumors (6). The expression of the miR-34 family of microRNAs is induced by p53, and the loss of miR-34a expression has been correlated with EMT through the induction of the EMT transcription factor SNAIL (7). Here, the authors show that IL-6 signaling, through its receptor IL-6R, activates STAT3, which directly represses MIR34A gene expression. Furthermore, repression of $\mathrm{miR}-34 \mathrm{a}$ was required for IL-6-mediated EMT, as miR-34a silences IL-6R (Figure 1). Finally, expression of this IL-6/STAT3/miR-34a feedback loop was discovered in primary colorectal tumors and associated with the presence of distant metastasis in patients.

The key experiment by Rokavec and colleagues (5) demonstrated that both IL-6 treatment and loss of miR-34a result in increased lung metastasis in a tailvein injection model of colorectal cancer compared with controls. Conceptually, if MET was not necessary for tumor formation at distal sites, then expression of miR-34a should remain low in the meta- 


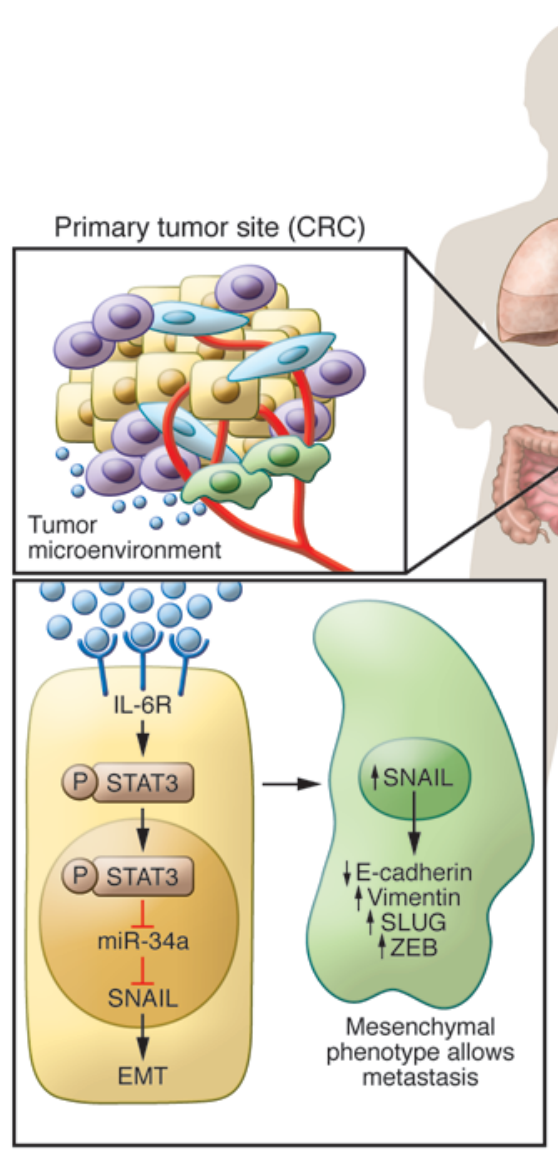

EMT

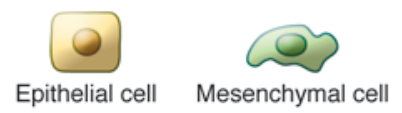

Epithelial cell Mesenchymal cell
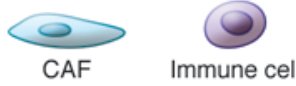

Metastatic tumor site (lungs)

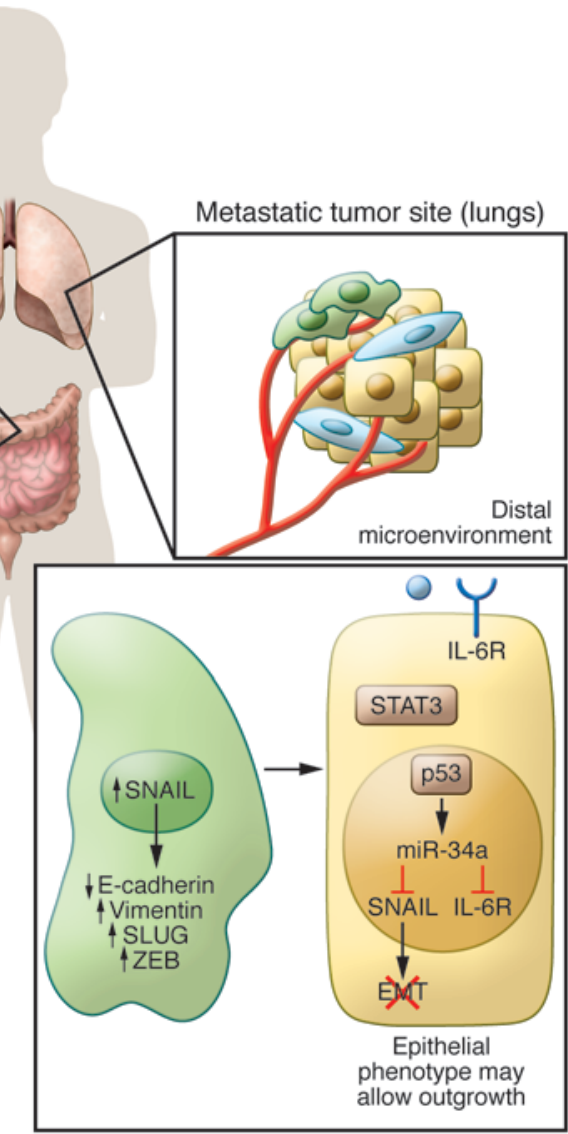

MET

CAF
Immune cell static lesions; however, Rokavec et al. (5) demonstrated that expression of miR-34a and other EMT markers was similar to non-IL-6-treated cells. These results suggest that activation of the IL-6R/STAT3/ miR-34a loop by IL-6-induced EMT shifts tumor cells toward a mesenchymal state that is advantageous for invasion, intravasation, and extravasation steps of the metastatic cascade. On the contrary, inactivation or disruption of the IL-6R/ STAT3/miR-34a loop, perhaps by the reduction of IL- 6 in a new microenvironment, induces MET and allows cells to switch back to an epithelial state. This shift could be important to facilitating colonization and outgrowth. Therefore, Rokavec and colleagues (5) have not only provided in vivo evidence for MET, but have also linked specific tumor microenvironments to activation and repression of a signaling loop that could explain the balance between EMT and MET.

\section{Is MET real in patients?}

As important as these findings are, many questions remain unanswered and need to be addressed in order to solidify the existence of MET in patients. For instance, the murine tail-vein injection model of colorectal cancer does not take into account the systemic effects of inflammation developed in response to the presence of a primary tumor. Specifically, the amount of IL- 6 in the primary microenvironment versus the distant microenvironment may not be different, especially considering that elevated IL- 6 in patient serum is often reported. Clinically, if IL-6 expression were elevated in the lung, would the size or epithelial characteristics of the metastases actually be decreased? Additionally, would not the presence of metastatic nodules also promote local inflammation and therefore start EMT all over again? Would this feedback loop be required or present if $\mathrm{p} 53$ function/miR34-a expression were completely ablated?

\section{Figure 1}

The IL-6/STAT3/miR-34a feedback loop is a molecular switch that controls EMT/MET transition. The complex milieu of primary colorectal cancer is mainly characterized by the presence of tumor epithelial cells, cancer-associated fibroblasts (CAF), vasculature, and cells of the immune system that interact and support each other in promoting tumor growth and local invasion. The inflammatory microenvironment, through its major mediator IL-6, triggers the activation of the IL-6R-mediated IL-6/STAT3 pathway, which represses the expression of miR-34a, thus inhibiting the miR-34a-mediated suppression of SNAIL. Therefore, the activation of the loop by IL-6-induced EMT, through its downstream effector SNAIL, shifts the cellular phenotype toward a mesenchymal state that is advantageous for the invasion, intravasation, and extravasation steps of the metastatic cascade. Conversely, the inactivation of this loop at the site of the metastatic colonization (i.e., lungs) aids in switching back to an epithelial state, namely MET, allowing for the outgrowth of metastatic nodules.
Most importantly, the implications for patient treatment still need to be determined. The association between inflammation and poor prognosis has already been established. Could the knowledge of this, and yet-to-be-determined molecular switches, be useful in developing treatments that could prevent the metastatic spread of tumor cells? These are difficult questions to answer due to the complex nature of the local and distant tumor microenvironments. However, now that a specific signaling pathway has been identified, autochthonous or syngeneic mouse models should be examined to determine the therapeutic utility of this pathway.

\section{Acknowledgments}

The authors would like to acknowledge their funding support, provided by NIH grants CA125550, CA155370, CA151925, DK081576, DK055001, and CA16672; 
the Metastasis Research Center at the MD Anderson Cancer Center; and the Cancer Prevention and Research Institute of Texas.

Address correspondence to: Raghu Kalluri, Department of Cancer Biology, University of Texas MD Anderson Cancer Center, 1515 Holcombe Blvd., Houston,
Texas 77054, USA. Phone: 713.792.8733; Fax:713.792.9636; E-mail: rkalluri@ mdanderson.org.

1. Kalluri R, Weinberg RA. The basics of epithelialmesenchymal transition. J Clin Invest. 2009; 119(6):1420-1428

2. Kalluri R, Zeisberg M. Fibroblasts in cancer. Nat Rev Cancer. 2006;6(5):392-401.

3. Grivennikov SI, Karin M. Inflammatory cytokines in cancer: tumour necrosis factor and interleukin 6 take the stage. Ann Rheum Dis. 2011;70(suppl 1):i104-i108. 4. Lopez-Novoa JM, Nieto MA. Inflammation and EMT: an alliance towards organ fibrosis and cancer progression. EMBO Mol Med. 2009;1(6-7):303-314.

5. Rokavec M, et al. IL-6R/STAT3/miR-34a feedback loop promotes EMT-mediated colorectal cancer invasion and metastasis. J Clin Invest. 2014:124(4):1853-1867.

6. Muller PA, Vousden KH. p53 mutations in cancer. Nat Cell Biol. 2013;15(1):2-8.

7. Hermeking H. p53 enters the microRNA world. Cancer Cell. 2007;12(5):414-418.

\title{
$\mathrm{Na}_{\mathrm{v}}$-igating through a complex landscape: SCN10A and cardiac conduction
}

\author{
David S. Park',2 and Glenn I. Fishman ${ }^{1}$ \\ ${ }^{1}$ Leon H. Charney Division of Cardiology and ${ }^{2}$ Heart Rhythm Center, New York University School of Medicine, New York, New York, USA.
}

\begin{abstract}
Genome-wide association studies (GWAS) have implicated SCN10A, which encodes a nociceptor-associated voltage-gated sodium channel subunit, as a modulator of cardiac conduction; however, this role has traditionally been ascribed to $S C N 5 A$, which is highly expressed in cardiac muscle. SCN10A is believed to affect cardiac conduction either directly through cardiomyocytes or indirectly via intracardiac neurons. In this issue of the JCI, van den Boogaard and colleagues introduce a third possibility: that the SCN1OA locus acts as an enhancer of $S C N 5 A$ gene expression. The authors demonstrate that $S C N 10 A$ expression is negligible within human and murine hearts, and that a T-box enhancer within the $S C N 10 A$ locus drives $S C N 5 A$ expression within cardiomyocytes. This work reasserts $S C N 5 A$ as the key determinant of cardiac conduction and highlights the importance of deciphering the functionality of coding versus noncoding regions when interpreting GWAS data.
\end{abstract}

\section{The unexpected finding: $S C N 10 A$}

Since the first identification of a significant association between SCN10A and ECG conduction parameters in genome-wide association studies (GWAS), a debate has emerged regarding how this gene and/or its product affect myocardial conduction (1-4). SCN10A encodes the $\alpha$ subunit of the voltage-gated sodium channel, $\mathrm{Na}_{\mathrm{v}} 1.8$, which was previously demonstrated to be expressed in nociceptive fibers of the dorsal root ganglia (DRG), but had never been implicated in heart physiology (5). Traditionally, rapid conduction properties of myocardial tissues have been ascribed to SCN5A, which encodes the pore-forming subunit of the major cardiac voltage-gated sodium channel, $\mathrm{Na}_{v} 1.5$. Mutations in SCN5A have been identified in patients with isolated cardiac conduction disease

Conflict of interest: The authors have declared that no conflict of interest exists.

Citation for this article: J Clin Invest. 2014; 124(4):1460-1462. doi:10.1172/JCI75240. and with the arrhythmic disorder Brugada syndrome $(6,7)$. In addition, mice with Scn5a haploinsufficiency exhibit slowed conduction parameters during cardiac electrophysiology testing (8). It was therefore unexpected that GWAS would more strongly correlate ECG parameters with SCN10A over SCN5A. This surprising finding raises the question of how $S C N 10 \mathrm{~A}$ fits into a landscape dominated by SCN5A. Furthermore, another GWAS has recently identified significant associations between Brugada syndrome and the SCN10A locus, making the link between SCN1OA and myocardial conduction slowing even more compelling (9).

\section{The cardiomyocyte hypothesis:} cell-autonomous role of $\mathrm{Na}_{\mathbf{v}} \mathbf{1 . 8}$

With the goal of establishing how SCN10A contributes to overall sodium current $\left(\mathrm{I}_{\mathrm{Na}}\right)$ in the heart, a combination of in vivo cardiac electrophysiology testing and cell culture-based biophysical analyses have been reported. Ambulatory telemetry monitoring was performed in wild-type mice treated with the $\mathrm{Na}_{\mathrm{v}} 1.8$-selective inhibitor, A-803467, as well as in Scn10a knockout mice, but the results were contradictory. Wild-type mice treated with A-803467 showed marked prolongation of PR and QRS intervals (1); however, Scn10a knockout mice exhibited shorter PR intervals with no change in QRS duration (4). These inconsistent results may represent differences between acute and chronic loss of $\mathrm{Na}_{\mathrm{v}} 1.8$, off-target effects of A-803467 on $\mathrm{Na}_{\mathrm{v}} 1.5$, or differential responses of cardiomyocytes and intracardiac neurons to A-803467. To elucidate the role of Scn10a specifically in cardiomyocytes, Yang et al. studied the effects of $\mathrm{Na}_{\mathrm{v}} 1.8$ inhibition or deletion in ventricular myocytes (10). Treatment of isolated mouse ventricular myocytes with A-803467 had no effect on peak $\mathrm{I}_{\mathrm{Na}}$, but blocked a component of the late sodium current, $\mathrm{I}_{\mathrm{Na}, \mathrm{L}}$, resulting in shortening of the action potential duration (APD) at slow stimulation frequencies. Consistent with the inhibitor studies, Scn10a- ventricular myocytes had reduced $\mathrm{I}_{\mathrm{Na}, \mathrm{L}}$, exhibited shorter APD at baseline, and were not responsive to A-803467 (10). Although these results suggest a role for Scn10a in APD prolongation and triggered arrhythmias, peak $\mathrm{I}_{\mathrm{Na}}$ and cardiac action potential upstroke velocity were unperturbed in ventricular myocytes with inhibition or deletion of $\mathrm{Na}_{\mathrm{v}} 1.8(10,11)$. Therefore, $\operatorname{Scn} 10 \mathrm{a}$ does indeed appear to have a cell-autonomous effect on cardiomyocyte electrophysiology, but the mechanism by which $\mathrm{Na}_{v} 1.8$ modulates cardiac conduction remained unclear. 